\title{
NeuroRegulation
}

\section{Treating Trauma Survivors with Neurofeedback: A Grounded Theory Study}

\author{
Christine L. Currie ${ }^{1^{*}}$, Theodore P. Remley, Jr. ${ }^{2}$, and Laurie Craigen ${ }^{3}$ \\ ${ }^{1}$ International Higher School of Social Work, Russian Orthodox Institute of Saint John the Divine, \\ Moscow, Russia, and New Hope Counseling Services, LLC \\ ${ }^{2}$ Our Lady of Holy Cross College, New Orleans, Louisiana, USA \\ ${ }^{3}$ Department of Counseling and Human Services, Old Dominion University, Norfolk, Virginia, USA
}

*Address correspondence to: Christine L. Currie PhD, LPC, NCC, P O Box \#179, Midland, Virginia 22728-0179, USA. Email: c.l.currie@verizon.net

Copyright: (c) 2014. Currie et al. This is an Open Access article distributed under the terms of the Creative Commons Attribution License (CC-BY).

\begin{abstract}
Neuroscience, the mental health field, and the concept of trauma as an underlying factor in mental and physical disorders have been inextricably linked since the inception of the mental health professions. Numerous quantitative studies have indicated that neurofeedback may be effective in ameliorating trauma symptoms; however, there is a paucity of research exploring the factors that produce those positive outcomes. The purpose of this qualitative grounded theory study was to explore the factors and processes that influence treatment outcomes when neurofeedback is used with trauma survivors. Thirty interviews were completed with 10 experienced mental health and neurofeedback professionals identified through a nomination process with a snowball sampling method. For this study a wide definition of trauma was used that included traumatic brain injury, the DSM-IV-TR (American Psychiatric Association, 2000) criteria for posttraumatic stress disorder, and the seven symptoms associated with complex trauma (Courtois, 2008; Courtois \& Ford, 2009; Herman, $1992,1997)$. Research results indicate that the neurofeedback practitioner is central to the treatment process, that practitioner therapeutic skills are crucial to positive neurofeedback outcomes, and that counseling and neurofeedback may effectively complement each other in trauma treatment.
\end{abstract}

Keywords: trauma; neurofeedback; grounded theory

\section{Background}

Neuroscience, the mental health field, and the concept of trauma as an underlying factor in mental and physical disorders have been inextricably linked since the inception of the mental health professions in the late 1800s, when Sigmund Freud and other physicians from the new professions of neurology and psychiatry studied hysteria. During World War I combat neurosis or shell shock was identified. In 1980, after the Vietnam War, the impact of trauma was officially recognized when posttraumatic stress disorder (PTSD) was included in the Diagnostic and Statistical Manual of Mental Disorders, III (DSM-III, American Psychiatric 
Association, 1980). Since the mid-1970s, the feminist movement has encouraged recognition of the adverse effects of interpersonal trauma (Herman, 1997), described in the construct of complex trauma, or CPTSD (Courtois, 2008; Herman, 1997).

On July 1, 2009, the Council for Accreditation of Counseling and Related Educational Programs (CACREP) officially recognized the importance of trauma when it mandated that disaster response, crisis intervention, and trauma knowledge be integrated into nearly every aspect of the counseling curriculum (CACREP, 2009). With the emphasis that CACREP has placed on trauma in the curriculum, it seems reasonable to investigate a wide variety of trauma treatments. Neurofeedback, based on recent neuroscience research, is one of those treatments.

Through neuroimaging techniques, neuroscience research has indicated that trauma affects the manner in which the brain receives and processes information (van der Kolk, 2006). Traumatic experience during the early developmental years may have an especially deleterious impact on all domains of functioning (Perry, 2002; Rothschild, 2000). Poor emotional regulation due to overarousal of the limbic system often results in limited problemsolving skills, inadequate relational abilities, and somatic symptoms (Courtois, 2008; van der Kolk, 2003).

With foundations in neuroscience, neurofeedback is based on the brain's plasticity that allows it to modify brain wave patterns in response to environmental changes (Charney, 2004). Neurofeedback is biofeedback applied to the brain and the central nervous system (Hammond, 2006). Numerous quantitative studies have indicated that neurofeedback may be effective in ameliorating symptoms often rooted in traumatic experience such as depression and anxiety (Hammond, 2005), substance abuse (Kelley, 1997; Peniston \& Kulkosky, 1999; Scott, Kaiser, Othmer \& Sideroff, 2005), posttraumatic stress disorder (Peniston \& Kulkosky, 1991; Smith, 2008), and somatic symptoms such as migraine headaches (Stokes \& Lappin, 2010) and fibromyalgia (Mueller, Donaldson, Nelson, \& Layman, 2001). However, there is a paucity of research exploring the factors that produce those positive outcomes. The purpose of this grounded theory study was to explore the factors and processes that influence outcomes when neurofeedback practitioners treat trauma survivors.

\section{Method}

Since the purpose of qualitative research is to discover rather than to test variables (Corbin \& Strauss, 2008), qualitative studies are appropriate when factors for quantitative research have not been fully identified. Grounded theory is a qualitative approach in which the researcher develops theory from fieldwork by exploring interacting concepts in a complex phenomenon. To be cohesive, one concept may be more prominent than the others as concepts interact in repeating action/interaction/emotional response patterns (Corbin \& Strauss, 2008).

\section{Participants}

Neurofeedback practitioners considered to be effective in treating trauma survivors were identified through a nomination process with a snowball sampling method. Participants consisted of seven women and three men whose ages ranged from the mid-50s to late 70 s. All were Caucasian. All were actively engaged in private practice in six different states representing four different regions of the United States, including large and medium 
metropolitan areas, a small college town, and rural towns of 3,000 to 4,000 people. The study included 3 participants who operate an integrated practice in which they divide their time more or less equally between neurofeedback and counseling services and 7 participants who offer primarily neurofeedback in their practices. Participants included licensed counselors, social workers, psychologists, and one practitioner certified in neurofeedback by the Biofeedback Certification International Alliance (BCIA).

Participants' years of experience in psychotherapy practice ranged from 14 to 40 years, with 25 years being the median. Experience in neurofeedback practice ranged from 4 to 21 years, with 15 years being the median. The number of clients seen per week ranged from 8 to 10 , to more than 100 , with 25 or more being the median. Between $25 \%$ and $95 \%$ of their clients have some form of trauma as an underlying issue, with $65 \%$ being the median.

Data collection took place in three rounds over a 9-month time frame. The first round was a face-to-face semi-structured interview, with one exception due to geographical limitations. The second round was a semi-structured telephone interview, and the third round was an email follow-up. To preserve confidentiality and anonymity, participants were given a pseudonym.

\section{Data Analysis}

The research team consisted of six students or recent graduates of the counseling doctoral program at Old Dominion University. All had completed training in qualitative research. Using a constant comparative method (Creswell, 2007), the research team analyzed the data and met on a regular basis to reach consensus on themes. The lead researcher transcribed the majority of the interviews. The initial open-coding process entailed analyzing individual participant's words or phrases, followed by axial coding in which themes were categorized across participants. Finally, using a selective coding process, the practitioner was identified as the prominent theme around which the other themes were organized (Corbin \& Strauss, 2008). The four other themes included: (a) the therapeutic process, (b) the neurofeedback process, (c) client factors, and (d) external factors. In order to triangulate the data (Patton, 2002), participants were given the opportunity to review their transcripts, read the analysis results, and provide feedback.

Although Creswell (2007) recommends bracketing assumptions, others hold that bracketing is impossible since assumptions and biases often have deep cultural roots difficult to identify (Corbin \& Strauss, 2008). Rather than bracket assumptions, I sought to use my experience to enhance the data gathering and analytic process by acknowledging my assumptions in an initial concept map (Strauss \& Corbin, 2008) that I shared with my research team before data collection began. I utilized a reflexive journal and regularly met with my research team and research partner to debrief, to reach consensus, and to modify the concept map. The Final Concept Map that was developed from the interviews is depicted in Figure 1. 


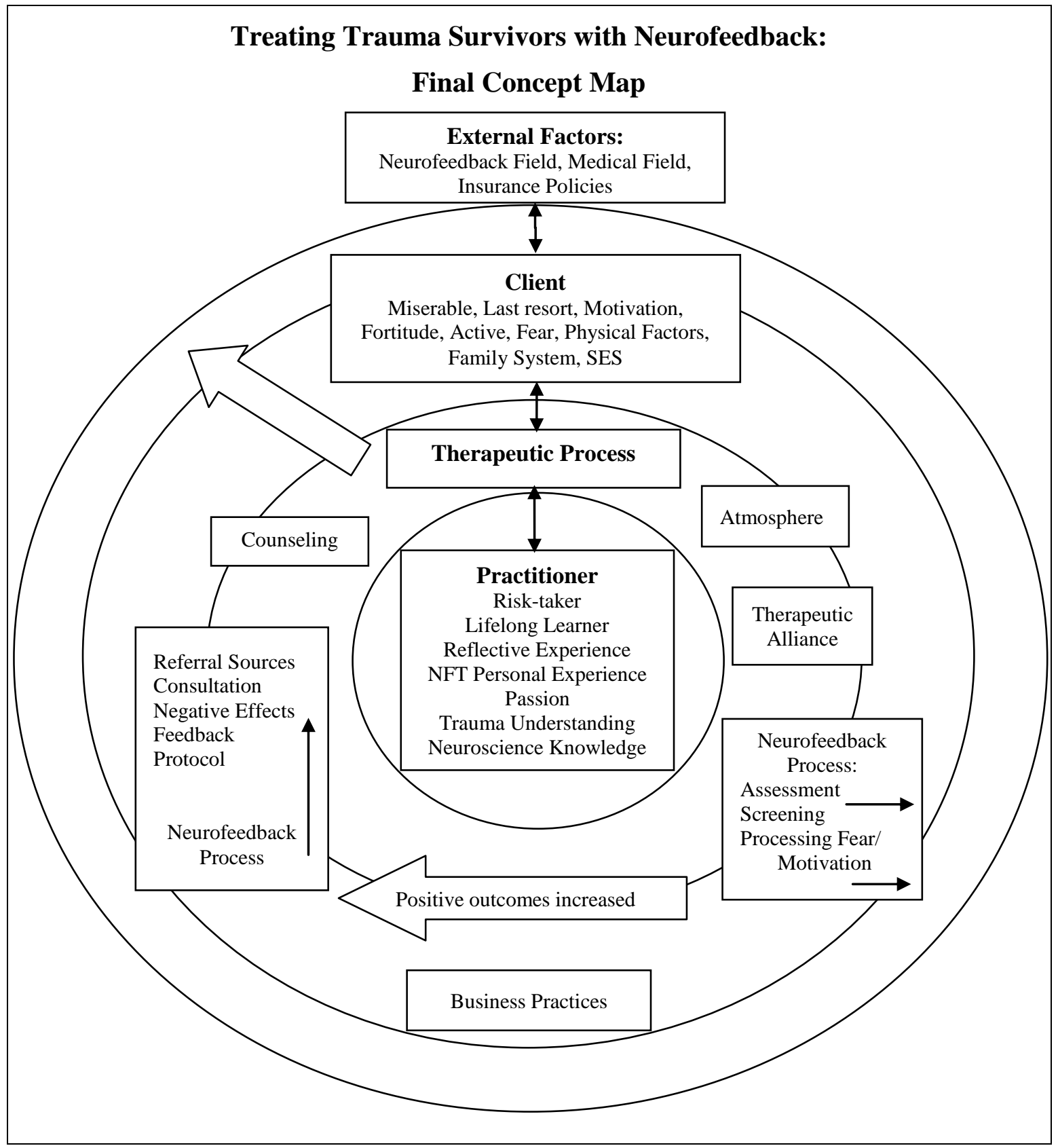

Figure 1. Final Concept Map. This was developed from the prominent themes that emerged from the three rounds of neurofeedback practitioner interviews. Research results indicate that the practitioner is central to the treatment process. Other themes include the therapeutic process, the neurofeedback process, client factors, and external factors.

\section{Trustworthiness}

Important criteria for determining the rigor of a qualitative study include the amount of researcher time spent in the field as well as the amount of data from which the researcher draws conclusions (Creswell, 2007). The data for this study consisted of 31 hours of 
recorded interviews in addition to a written email follow-up. I spent approximately 40 additional hours in the field with participants in informal ways. In addition, I attended a 4-day neurofeedback training before data collection began and a 1-day conference during the study. The external auditor for this study examined the audit trail, which consisted of 1,290 pages of materials.

\section{Results}

The central research question for this study was, "What are the factors and processes that influence treatment outcomes when neurofeedback is used to treat individuals with trauma symptoms?" Five major themes, each with subthemes, emerged from the data. The central theme is the practitioner.

\section{Theme 1: The Practitioner: "The Magic is Not in the Box"}

The practitioners in this study described themselves as being "open" to new ideas and experiences, and as frequently thinking "out of the box." They entered the mental health field because they are people-oriented and relational, but then made the choice to tackle technological challenges that they describe as being "daunting," "a huge leap," "a steep/huge/enormous learning curve," and a "step of faith." They described "sweating blood" and being "scared to death" when they incorporated neurofeedback into their practice. Gaylen mortgaged her house to purchase her first neurofeedback set. All were willing to take the "leap," to undergo a "huge paradigm shift" because what they saw before them was so "dramatic" and "compelling" that they could not "walk away." They are risk-takers who embrace new challenges.

Subtheme 1.1: Risk-takers. The risk-taking continues throughout the neurofeedback process. Practitioners stated that neurofeedback could be "scary" for both practitioner and client, as negative effects may happen unexpectedly. Trauma survivors may feel more "vulnerable" as they lose their "protective armor" or as flashbacks of traumatic events suddenly occur. Barbara described the strong inner fears of the therapist in the face of uncertain treatment results:

I have to always stay calm with clients ... and if there is a problem to always reassure your client that everything's going to be fine because there's that placebo effect that takes place, even if you're scared to death inside and wondering, "Oh no, what did I do and how am I going to fix this?" (Barbara)

Marian routinely expects minor side effects that indicate that neurofeedback results are not merely from a placebo effect.

A lot of people say this tiny signal can't be having an effect so it has to be a placebo effect, but I'm convinced it's not because the most common reaction ... you will have a headache or feel really tired or re-experience the pain at the time of trauma with the first treatment or two ... so negative things happen ... if they have ever had a concussion, they are likely to have a headache ... because there is a recall of the somatic experience.

Marian emphasized that clinicians must be "unflappable" in working with trauma survivors. 
Subtheme 1.2: Lifelong learners: "Always looking." These expert practitioners seem to possess an inner drive to surpass mere competence in order to be the best that they can be in their unique settings.

I think experts are always looking ... the people that I think of as the best [emphasis] ... are always looking for new approaches ... they are talking to colleagues ... when they get stuck, they are talking ... they are finding other people who are at their level of experience or that they trust. (Gaylen)

As a result of "always looking," these practitioners have accumulated a "big bag of tools" (Sarah) and a "whole array in my armament to help people" (Julia) that may include play therapy, Eye Movement Desensitization and Reprocessing (EMDR), Dialectical Behavior Therapy (DBT), psychodrama, guided imagery, or hypnosis. Some utilize biofeedback along with neurofeedback to access the autonomic nervous system as well as the central nervous system. They often utilize multiple types of equipment. No matter what skills they already possess and what equipment they currently use, they are continually striving to improve.

What I really want to do is keep learning and stay on the cutting edge ... I don't want to coast ... I want to always be one of the best ... If I'm offering something to my clients, I want to be able to offer them the best that's out there ... I'm always a little behind because there's always a learning curve ... but I ... keep working and keep learning. (Barbara)

Staying on the "cutting edge" in order to offer clients "the best that's out there" requires dedication to continuing education. Most of the participants cited ongoing training as one of the most important factors for positive client outcomes. Julia stopped counting her continuing education units after she surpassed 1,000 post-doctorate hours. Participants attend national conferences and local trainings, participate in email list serves, and consult regularly with colleagues. Their conversations are interspersed with references to seminars, books, and professional journal articles. Most have had at least one strong mentor. In addition, most participants have published in professional journals. Some have researched their own practices in order to assess their effectiveness. All these activities result in consistent professional growth:

I'm a better clinician than I was a year ago-and I'm certainly a heck of a lot better than I was 10 years ago—but every year... I am better than the year before. (Barbara)

In addition to investing in themselves, the participants invest in others and in the field as a whole. They serve on the boards of their professional associations, organize local trainings for other professionals, and present at conferences. They mentor newer colleagues both formally and informally by taking on interns in their practices and through informal consultation.

Subtheme 1.3: Reflective experience. Participants counted their years of experience as being vastly important in client outcomes. When asked how much experience matters, most simply answered "a lot." Experience gives "more information to draw from ... it just pops right out when you need it" (Shirley). Experience helps practitioners to "recognize

phenotypes" (Julia) and to handle abreactions. It encourages practitioners to keep searching for new protocols as they realize the limits of what they currently know. In addition, experience is not measured only in years, but in reflection on their varied experiences. 
Practitioners discussed their disappointments and mistakes as well as their successes, and what they learned from both.

Subtheme 1.4: Neurofeedback personal experience. Six practitioners in this study experienced dramatic physical and emotional changes from neurofeedback training. Others were convinced through seeing rapid changes in clients or family members.

Gaylen describes herself as a "poster child for everything neurofeedback can address." She had PTSD and a traumatic brain injury (TBI) as a result of childhood trauma until neurofeedback training eliminated her seizures, migraine headaches, and all PTSD symptoms, including her life-long startle response. Julia had spent "tens of thousands of dollars" seeking a cure from chronic fatigue syndrome and envisioned founding a "healing center" if she could ever recover. After neurofeedback she founded and manages one of the largest clinics in the country. Marian had struggled her entire life with memory and learning problems stemming from a childhood TBI. After neurofeedback training, "it all changed ... it changed my life ... I couldn't be doing this [the research] if I didn't have that treatment." She says, "Nothing else does this." After Helen witnessed "profound" mood changes in her son due to neurofeedback training, she also began treatment and was cured of chronic fatigue syndrome. She currently manages a clinic and travels nationally and internationally to train others. David was cured of "30 years of back pain" in a neurofeedback session. Barbara "made more progress in 3 months of neurofeedback than in 20 years of counseling" for anxiety and panic disorder.

Subtheme 1.5: Passion. Risk-taking, lifelong learning with motivation to better serve clients, combined with their own personal experiences of neurofeedback, produces a passion for the field and a vision for its future.

Just like everybody who gets into this field now, it's like, "Oh my God, have you thought of all the people you could be helping?" Let's get busy; let's make this happen in the world. (Helen)

And these practitioners are making neurofeedback "happen in the world" in creative ways. Sarah has a plan to attract interns to her underserved area. Gaylen has trained mental health professionals to use neurofeedback with abused children in a children's clinic. Charles carries portable neurofeedback equipment to clients' homes if they are housebound. Marian recruits veterans for TBI and PTSD studies. Helen's practice sponsored a research study at a residential treatment facility for drug and alcohol addicted individuals. David treats military veterans free of charge, saying, "Money doesn't stand in the way around here." Several mentioned at times working either pro bono or on a sliding scale. Helen stated, "It's not work; it's a passion."

Subtheme 1.6: Trauma understanding. Participants agreed that trauma is a common experience that takes many forms, including PTSD, complex trauma, and TBI. However, clients may not realize that the underlying source of their symptoms is trauma. David noted that "half or less" of his clients come to therapy for the actual underlying problem, "So we have to figure it out." Practitioners emphasized going slowly with trauma survivors due to potential abreactions and that trauma survivors need counseling in addition to neurofeedback.

Complex trauma, often rooted in early childhood abuse or neglect, causes disruptions in "every system of cognitive, learning, behavioral, emotional, and physiological [domains]." 
With trauma survivors Gaylen focuses on "always to be quieting fear." Julia described complex trauma as having been "marinated in fear," resulting in a "body clench" as if a person has been "holding onto something" for a long time. If a client has many somatic complaints, including migraine headaches or chronic upsets such as irritable bowel syndrome, or emotionally seems "paralyzed in life," then Julia suspects trauma as an underlying factor. Helen described complex trauma survivors as having "no place of safety" within themselves, such that they feel overwhelmed and constantly threatened. Addictions and other self-destructive behaviors may result from the feeling of low self-worth that often accompanies trauma. Sarah stated that underlying trauma issues in children are often misdiagnosed as AD/HD or some other disorder. Childhood trauma causes a "coping deficit" because the prefrontal cortex cannot develop when a child is continually in a "survival mode." Complex trauma can take many forms.

Marian's definition of trauma includes all degrees of TBI, including Cesarean sections and other "traumas that aren't usually thought of as traumas, but they're a trauma for the brain." She assesses carefully for any type of head injury because even mild head injuries can dramatically alter emotional responses and thinking processes.

Subtheme 1.7: Neuroscience knowledge. Participants discussed neurofeedback treatment protocols in terms of neuroscience knowledge. Sarah discussed the inability of the prefrontal cortex to develop normally in a child exposed to ongoing trauma. Gaylen described how "the brain becomes mind," meaning that personality is formed through the underlying firing of brain neurons. She does not utilize cathartic therapies due to the maxim "what fires together wires together." Discussing traumatic events before the brain is calmed down only serves to strengthen the neuronal pathways in an anxious, fear-based neurological system. Marian described the "neurochemistry of trauma" as the brain being "stuck," being "frozen in a protective state" from "extreme fear."

Fear may cause the brain to be stuck, but neurofeedback can help it get unstuck by modifying brain-firing patterns. Marian emphasized that neuroplasticity, defined as "the brain's ability to change," is a practitioner's "biggest ally" in neurofeedback treatment:

One thing I learned when I was working with hospice and cancer patients who were dying ... is that there is something in every cell that keeps trying to fix itself, and the brain is just sitting there waiting for us to find the right key ... neuroplasticity is the thing.

Important factors in successful treatment involve the neuroplasticity of the client's brain coupled with the practitioner's ability to find "the right key" based on neuroscience knowledge.

\section{Theme 2: Therapeutic Process}

The therapeutic process begins with the office atmosphere, continues with the formation of the therapeutic alliance, and builds as the therapist responds to client feedback during every session. Neurofeedback is a major piece, but actually only one piece of this holistic process.

Subtheme 2.1: Atmosphere. The therapeutic process begins the moment a client enters the door. Barbara deliberately sets up a "friendly kind of atmosphere." David spoke of the importance of a "loving atmosphere," a "sense of love and God's presence" that "sets up the possibility for healing." Sarah's office, located in a renovated church with stained glass 
windows, sets up a "safe, welcoming" environment that is a "huge factor" in therapeutic outcomes. The walls of Julia's waiting room are decorated with enlarged newspaper articles of neurofeedback success stories. A notebook filled with research articles and more success stories sits prominently on a table in the center of the room. I found myself wanting to be a client as I read these materials, and I wondered what degree of hope and confidence might be instilled in clients even before the actual neurofeedback process begins.

Subtheme 2.2: Therapeutic alliance. The office atmosphere provides the prelude to the formation of the therapeutic alliance, which grows over time and is particularly fragile with trauma survivors (Gaylen). In his 40 years of counseling experience, David's most important lesson has been the centrality of empathy, an ability to "hear" clients, and to "feel a sense of what they feel." The practitioner may be like a "coach" or a "support person" (Helen). "Trust" within a "nurturing relationship" is important (Barbara). A caring relationship is crucial because "anecdotally we learned that people are not used to being treated with care" (Sarah). Having military clients, Marian sat through the movie Hurt Locker even when she wanted to walk out, thinking, "These guys live [emphasis] this so surely I can watch a movie." It is important to "be there" as a strong, stable, and calm support for trauma survivors (Barbara). Even in the midst of complicated technological equipment, David summed up the importance of the relationship in the neurofeedback process by saying, "I am not a technician ... I am still a therapist."

Subtheme 2.3: Business practices. Business practices constitute the foundation on which practitioners build the therapeutic process with clients. Marian observed that one prominent organization, now out of business due to poor decisions, no longer exists to train people. Practitioners discussed careful scheduling to "stay afloat" and equipment prices and sales. Julia's marketing expertise shows in her ability to attract attention from the press. Helen markets her practice by offering a free first appointment in which she familiarizes people with neurofeedback. Without a savvy business sense, practitioners could not be helping anyone.

\section{Theme 3: Neurofeedback Process: "We're Not Fixing Brain Waves”}

Neurofeedback is more than "fixing brain waves" (Helen). It sets in motion a personality "transformational process" as the practitioner works at the intersection of the brain and the mind.

The most important lesson that I've gotten has been that the core of all of our psychological problems rests in the firing of the brain in some way, ... that all mental processes sit on top of this and that we have access to it.... In neurofeedback you see an evolution of ... the mind as the brain regulates itself. (Gaylen)

Subtheme 3.1: Thorough assessment: "Detective agency," "Outside the box." This "transformational process" starts with a thorough assessment and screening. Marian likened the assessment to being a "detective agency" in which practitioners look "outside the box" for the root problem. Marian screens for head injuries, Lyme disease and other parasitic infections, sometimes the root of psychological and emotional problems. She assesses for trauma by asking, "What's the worst thing that has ever happened to you?" Clients may be sent for nutritional counsel or allergy testing, and may be told to stop ingesting aspartame, a neurological toxin.

Subtheme 3.2: Screening: “Don’t waste your money with me!” Either in conjunction with the assessment or separately, many practitioners utilize some type of screening process. 
Julia is very straightforward with clients in saying, "Don't waste your money with me!" Clients are screened for chronic infections, some addictions, and possibly benzodiazepine medications that "take over the brain." Practitioners may also assess client motivation. Through "good, careful informed consent," Julia and Shirley stress to clients that neurofeedback is "not an instant fix." David asks clients, "What do you have to give up?" in order to get better. Gaylen asks, "You can go through a wide range of changes. Are you ready for that?" Both Sarah and Gaylen highlight the importance of processing fear since "health is scary sometimes."

Subtheme 3.3: Protocol: "Cast a wide net," "Find the right key," "Stabilize." With trauma survivors, practitioners move cautiously to "calm down" and "stabilize." Julia talked of "casting a wide net" and having "a whole array in my armament to help people." Participants often utilize multiple techniques because "good therapists have always been very attuned to what people bring, and so they wouldn't necessarily be wedded to one approach" (Marian). Practitioners "find the right key" in order to talk to the brain and not at the brain (Marian).

Subtheme 3.4: Constant feedback: "The brain and the mind." Practitioners choose protocols as they follow client symptoms: "Start with the symptom or the behavior that is most obvious; that's going to be the brain's way of talking to you, the outsider" (Marian). Protocols are altered in response to specific client feedback every session. Helen describes this process as "a team effort" in which she tells clients, "You and I are going to have to work together here ... I kind of know about neurofeedback, but do you know about you? So you've got to talk to me about what's going on." When receiving client feedback, the practitioner is having "two different conversations" simultaneously with both "the brain and the mind." For example, if a client reports that he is calmer (the mind), but is having nightmares of killing people (the brain), the practitioner follows what the over-aroused brain is saying and institutes more calming protocols (Gaylen). Lastly, participants agreed that effective neurofeedback treatment involves art or tuition as well as science. "Intuitive sensing" (Charles) and a "sense of timing" (Sarah) help the practitioner to "apply the model in a sensitive way for the individual" (Helen).

Subtheme 3.5: Negative effects. Although potentially "scary" for both the client and the practitioner, negative reactions may indicate that neurofeedback results are not merely "placebo effect," as some detractors claim. Negative effects can actually increase client hope in the process.

Even when we move people in the wrong direction ... even when we mess up their sleep or give them a headache or something ... that gets people's attention ... because the biggest fear is really that this is a scam ... you're wasting my time. And when we do something strong, even if it's the wrong thing, it's like, "Ok, this actually works" because after all, what is people's experience with medicines? They just as often mess you up as help you ... this is strong stuff and if I get the right strong stuff this may help me. (Helen)

Subtheme 3.6: Consultation and referral sources: “I don't know it all!" All participants emphasized the importance of consulting with colleagues. The statement, "I don't know it all!" spoken by a practitioner with over 31 years of mental health experience and over 15 years of neurofeedback experience, exemplifies the importance placed on consultation.

Subtheme 3.7: Counseling: "Who am I now?” Counseling and neurofeedback complement each other. Some likened counseling trauma survivors without neurofeedback 
to "doing surgery without anesthesia." Gaylen will not counsel trauma survivors without neurofeedback as a calming component. Particularly with complex trauma survivors, the counseling process can be hindered by transference reactions of fear, rage, and shame, accompanied by therapist countertransference. Neurofeedback provides the "transference cure" by "quieting fear" in the limbic system so that the client can discuss trauma without disregulating the neurological system. Neurofeedback helps to protect the therapist from vicarious trauma.

Neurofeedback calms the trauma survivor's anxious neurological system, and counseling assists in processing the changes that neurofeedback may effect in every domain. Since "whole identities may be built around affect," as affect symptoms drop away, the question of identity comes to the forefront. Clients may begin to ask themselves, "Who am I? If I am not this terrified, raging, shame-based person, who am I?" (Gaylen). In neuroscience terms, neurofeedback calms the brain and makes it more flexible. Talking within the counseling relationship helps to move the traumatic experience from the more emotional right brain to the more linear, rational left brain, so that the client can be "done with" the trauma. The client goes back and forth between the emotion regulation that neurofeedback provides and the talking in the counseling relationship that assists the client in identity transformation as affect symptoms drop away.

\section{Theme 4: Client Factors}

Neurofeedback clients are generally "miserable," have "tried everything else," and then try neurofeedback as a "last resort." In addition to nutrition, allergies, toxins, and parasitic infections, participants identified the following client factors as potentially affecting treatment outcomes.

Subtheme 4.1: Motivation. Client motivation, described by Sarah as, "You gotta [sic] have a want to," is foundational. Even if motivated at the beginning of treatment, clients need "fortitude" to stay with the process sometimes over significant periods of time. Moreover, clients must be willing to process and overcome fear of health.

Subtheme 4.2: Family system. A psychologically toxic environment will likely interfere with treatment outcomes. If a client is a "designated patient" that is "holding the family together," if the family does not want to "lose their scapegoat," or if a client has an unstable or abusive home life, neurofeedback effects will likely be diminished.

Subtheme 4.3: Multicultural factors. All 10 participants maintained that, since neurofeedback is not as "culturally loaded" as traditional talk therapy, it tends to cross-racial and ethnic boundaries well. Participants specifically noted that "age is not a factor," as they have successfully treated clients ranging from 3 months to 96 years. Although more women than men come for neurofeedback, both respond well. The one multicultural factor that does affect the process is socioeconomic status. A client must have sufficient financial resources to enter neurofeedback treatment initially and to stay engaged over time.

\section{Theme 5: External Factors}

External factors are defined as factors outside the therapist, the client, and the therapeutic process that practitioners identified as influencing neurofeedback treatment outcomes. 
Subtheme 5.1: The neurofeedback field. Participants described the neurofeedback field as being "cutting edge," "growing exponentially," "up and coming," and having "endless possibilities." They were enthusiastic about research published in professional journals as well as recent publicity on radio broadcasts, the New York Times, and other public forums. Acceptance of neurofeedback as a viable treatment option may be reaching a "critical mass," particularly as people become concerned with medication side effects, especially in children.

All publicity is not positive, however. Since neurofeedback is a relatively new field whose professional identity is unclear, a credentialing process has not yet been standardized. As a result there are "a lot of quacks out there" who may bring bad publicity to the field at a time that it is struggling to earn its place in the mainstream. Both good and bad publicity potentially affect client confidence as well as neurofeedback credibility with medical professionals.

Subtheme 5.2: The medical field. The medical field was also viewed as exerting both a positive and negative influence on neurofeedback outcomes. On the positive side, psychiatrists and other medical professionals are beginning to attend trainings, refer clients, and coordinate treatment with neurofeedback providers. Increased acceptance by medical professionals moves neurofeedback out of the "fringe" and "experimental" realm to a more respected place in the mainstream. This acceptance may encourage clients to initiate and to stay with the process.

On the other hand, the medical field was also viewed as perpetuating the overall "culture of medication" that encourages people to "take a pill and be fixed." Clients with this mentality are less likely to invest the time and finances necessary for successful neurofeedback treatment. Those who do seek neurofeedback are sometimes discouraged by physicians who tell them that they are "wasting their money" on a "ridiculous" treatment that "won't do anything." Some clients resist physician negativity, some change physicians, but others drop out of treatment.

Subtheme 5.3: Insurance policies. Closely related to the medical field are insurance policies that sometimes prohibit reimbursement for neurofeedback services. Clients lacking the financial means to initiate or continue the process are excluded from its positive effects.

\section{Discussion}

Findings of this study indicate that the practitioner is central to neurofeedback treatment outcomes with trauma survivors. Other themes included the therapeutic process, the neurofeedback process, client factors, and external factors. A comparison of these findings with existent literature follows.

The therapist has been the focus of numerous studies, as research has indicated that therapist competence is crucial for successful results (Ronnestad \& Skovholt, 2003). Jennings and Skovholt (1999) described cognitive, emotional, and relational (CER) characteristics of master therapists that constitute a three-legged stool of expertise. In the cognitive domain master therapists are voracious learners, use experience as a major resource, and value "cognitive complexity and the ambiguity of the human condition" (p. 6). In the emotional domain they are "self-aware, reflective, nondefensive, and open to feedback" as well as "mentally healthy and mature individuals" (p. 7) who take care of themselves. In the relational domain they value the importance of the therapeutic alliance and are comfortable with clients' intense emotions. 
Consistent with the findings of Jennings and Skovholt (1999), in the cognitive domain participants in this study were lifelong learners "always looking" for better treatment options. They valued reflexive experience and embraced complexity in looking "outside the box" for root causes. In the emotional domain they were receptive both to client feedback and to colleague consultation. Many maintained their own mental health by engaging in neurofeedback training and other self-care activities. In the relational domain they valued the therapeutic alliance as an essential overarching piece of the neurofeedback process. They emphasized listening, caring for clients, processing fears and being "unflappable" in the face of client emotions. It would seem that effective neurofeedback practitioners, despite the addition of technological equipment to the therapeutic process, are master therapists as well as neurofeedback specialists.

Wounded healers are individuals whose personal experience of suffering is transformed into a constructive healing force for fellow sufferers. The theme of the wounded healer is found in literature across time and cultures, and in both the medical and mental health literature (Jackson, 2001). It surfaces in this study as 7 of the 10 participants alluded to their own personal journeys through various forms of emotional trauma or physical illness or both. Perhaps one participant's comment best describes the wounded healer: "We try it on ourselves first, and then what works, we pass along."

Asay and Lambert (1999) estimated that $15 \%$ of treatment outcome is due to expectancy or placebo effects, sometimes called the hope factor. Participants engender client hope through their office atmosphere, through staying calm in unexpected client reactions, and in generally "being there" for clients in a strong therapeutic relationship. Even negative effects may actually increase hope that the neurofeedback is working. David unabashedly stated, "Placebo works!"

Results of this study indicated that the therapeutic alliance is an essential ingredient to positive neurofeedback outcomes as it is crucial to successful psychotherapy (Horvath \& Symonds, 1991; Sexton \& Whiston, 1994). According to the common factors model, 30\% of client improvement depends on the therapeutic alliance (Asay \& Lambert, 1999). Although this study did not seek to quantify the amount of influence the therapeutic alliance exerts on treatment outcomes, participants were clear that the therapeutic relationship is important.

Findings in this study differed from the common factors model (Asay \& Lambert, 1999) in several ways. According to Asay and Lambert (1999), only $15 \%$ of psychotherapy outcomes are derived from actual techniques, which are more or less interchangeable. In contrast to these findings, participants in this study emphasized the necessity of having a "big bag of tools" and "a whole array in my armament to help people." For the most part, participants viewed the ability to change neurofeedback treatment protocols as an essential ingredient in successful outcomes. In addition, findings of this study emphasize the therapist as being central to the process, in contrast to the Asay and Lambert (1999) model that de-emphasizes the therapist role. It may be that since neurofeedback therapy requires more technological knowledge, the therapist is more central to the neurofeedback process than to the psychotherapy process.

Research participants in this study concurred that client factors as well as therapist factors contribute to treatment outcomes. Asay and Lambert (1999) assert that as much as $40 \%$ of client improvement derives from extra-therapeutic factors related to the client. These client factors may include the nature of the problem, motivation, ability to relate, ego strength, and 
family and social support. Participants in this study identified motivation, the family system, and the nature of the problem as potential client factors that contribute to treatment outcomes.

The research participants' perception that multicultural factors exert little or no influence on neurofeedback treatment outcomes, with the exception of socioeconomic status, is congruent with the neurofeedback literature. Although few specifically multicultural studies exist, one study has indicated that neurofeedback can be adjusted in a culturally sensitive manner with positive treatment outcomes in a Native American population (Kelley, 1997). Studies have also indicated successful neurofeedback results with groups composed of both males and females and those representing a range of ages (Scott et al., 2005). Drawing from the responses of the study participants as well as from neurofeedback research, it seems that neurofeedback may be a treatment option in a wide range of cultural situations. The challenge is creating a conduit for neurofeedback services to be brought to these diverse populations.

\section{Limitations}

Several limitations to the findings of this study should be noted. Since snowball sampling seeks information-rich informants rather than a representative participant sample, the findings of this study cannot be generalized beyond this group, and the voices of some highly regarded practitioners may have been excluded. Furthermore, the lack of diversity in the participant sample may have skewed themes in ways unknown at this time. Lastly, this article is a condensed version of a larger study. Due to its condensed form, participant profiles and other information that would have provided a fuller context for the themes could not be included.

\section{Implications}

The results of this research study have several implications for counselors and counselor educators. First, although none of the neurofeedback practitioners in this study advertise themselves as specializing in trauma, between $25 \%$ and $95 \%$ of their clients have some form of trauma as an underlying issue, with $65 \%$ being the median. With such a significant portion of clients being trauma survivors, it would seem that CACREP has made a strategic decision to improve counselor competence by incorporating trauma education into the counseling curriculum (CACREP, 2009).

Secondly, several practitioners highlighted the fact that clients may engage in psychotherapy for years with minimal improvement because the root cause of the difficulty is actually an undiagnosed head trauma or an underlying infection. Teaching on the impact of physical root causes of mental disorders, instruction on how to conduct a holistic assessment as well as on the importance of appropriate referral sources could be a beneficial component to mental health education.

Lastly, since findings from this study indicate that neurofeedback may be an effective complement to counseling with trauma survivors, it would be beneficial for counselors to be familiar with this treatment option, whether or not they decide to incorporate neurofeedback into their practices. Being knowledgeable about the manner in which a counselor and a neurofeedback practitioner can work together may hold significant potential for increasing positive client outcomes. Some counselor education programs have begun offering a track 
that leads to biofeedback and neurofeedback certification by the Biofeedback Certification International Alliance (BCIA). More universities could consider offering this track.

\section{Future Research}

The findings of this study indicate that neurofeedback is an effective treatment modality for trauma symptoms and that the neurofeedback practitioner plays a central role in treatment outcomes. Future research may expand on the themes highlighted in this study.

Since the themes in this study were drawn exclusively from the practitioner's point of view, the central role of the practitioner may be overemphasized and the importance of client factors may be underemphasized. A future qualitative study from the trauma survivor's perspective might provide a more balanced view of the process.

One study participant suggested that more research could be completed in the neurofeedback field to specifically answer the question, "When neurofeedback doesn't work, why doesn't it work?" In addition, studies comparing treatment protocols for all the various types of trauma would add to the body of research already in existence.

One salient characteristic of the effective therapists in this study was the value that they placed on continuing education and "always looking" for better treatment options. Further research may provide more understanding of the development of master therapists and effective neurofeedback practitioners. Is there a way to more effectively inspire this motivation for excellence within our mental health training programs and beyond?

\section{References}

American Psychiatric Association. (1980). Diagnostic and statistical manual of mental disorders ( $3^{\text {rd }}$ ed.). Washington, DC: Author.

American Psychiatric Association. (2000). Diagnostic and statistical manual of mental disorders ( $4^{\text {th }}$ ed., text rev.). Washington, D.C.: Author.

Asay, T. P., \& Lambert, M. J. (1999). The empirical case for the common factors in therapy: Quantitative findings. In M. A. Hubble, B. L. Duncan, \& S. D. Miller (Eds.). The heart and soul of change: What works in therapy ( $1^{\text {st }}$ ed., pp. 23-55). Washington, DC: American Psychological Association.

Charney, D. S. (2004). Psychobiological mechanisms of resilience and vulnerability: implications for successful adaptation to extreme stress. The American Journal of Psychiatry, 161, 195-216. http://dx.doi.org/10.1176/appi.ajp.161.2.195

Corbin, J., \& Strauss, A. (2008). Basics of qualitative research: Techniques and procedures for developing grounded theory ( ${ }^{\text {rd }}$ ed.). Thousand Oaks, CA: Sage.

Council for Accreditation of Counseling and Related Educational Programs, 2009 Standards. Retrieved from http://www.cacrep.org 


\section{NeuroRegulation}

Courtois, C. A. (2008). Complex trauma, complex reactions: Assessment and treatment. Psychological Trauma: Theory, Research, Practice, and Policy, S, 86-100. http://dx.doi.org/10.1037/1942-9681.s.1.86

Courtois, C. A., \& Ford, J. D. (Eds.). (2009). Treating complex traumatic stress disorders: An evidence-based guide. New York: Guilford Press.

Creswell, J. W. (2007). Qualitative inquiry and research design: Choosing among five approaches (2nd ed.). Thousand Oaks CA: Sage Publications.

Hammond, C. (2005). Neurofeedback treatment of depression and anxiety. Journal of Adult Development, 12, 131-137. http://dx.doi.org/10.1007/s10804-005-7029-5

Hammond, D. C. (2006). What is neurofeedback? International Society for Neurofeedback \& Research. Retrieved from http://www.isnr.org/uploads/whatisnf.pdf

Herman, J. (1992). Trauma and recovery. New York: Basic Books.

Herman, J. (1997). Trauma and recovery: The aftermath of violence-from domestic abuse to political terror. New York: Basic Books.

Horvath, A. O., \& Symonds, B. D. (1991). Relation between working alliance and outcome in psychotherapy: A meta-analysis. Journal of Counseling Psychology, 38(2), 139-149. http://dx.doi.org/10.1037/0022-0167.38.2.139

Jackson, S. W. (2001). Presidential address: The wounded healer. Bulletin of the History of Medicine, 75, 1-36. http://dx.doi.org/10.1353/bhm.2001.0025

Jennings, L., \& Skovholt, T. M. (1999). The cognitive, emotional, and relational characteristics of master therapists. Journal of Counseling Psychology, 46, 3-11. http://dx.doi.org/10.1037/0022-0167.46.1.3

Kelley, M. J. (1997). Native Americans, neurofeedback, and substance abuse theory: Three year outcome of alpha/theta neurofeedback training in the treatment of problem drinking among Dine (Navajo) people. Journal of Neurotherapy, 2(3), 24-60. http://dx.doi.org/10.1300/J184v02n03 034

Mueller, H. H., Donaldson, C. C. S., Nelson, D. V., \& Layman, M. (2001). Treatment of fibromyalgia incorporating EEG-driven stimulation: A clinical outcomes study. Journal of Clinical Psychology, 57, 933-952. http://dx.doi.org/10.1002/jclp.1060

Patton, M. Q. (2002). Qualitative research and evaluation methods (3rd ed.). Thousand Oaks, CA: Sage Publications.

Peniston, E. G., \& Kulkosky, P. J. (1991). Alpha-theta brainwave neuro-feedback therapy for Vietnam veterans with combat-related post-traumatic stress disorder. Medical Psychotherapy, 4, 47-60.

Peniston, E. G., \& Kulkosky, P. J. (1999). Neurofeedback in the treatment of addictive disorders. In J. R. Evans \& A. Abarbanel (Eds.), Introduction to quantitative EEG and neurofeedback. (pp. 157-179). San Diego, CA US: Academic Press. 


\section{NeuroRegulation}

Perry, B. (2002). Brain structure and function II: Special topics informing work with maltreated children. Retrieved from http://www.harmonyadoptions.org/uploads/pdf/Brain\%20Structure\%20and\%20Functi on\%20-\%20Maltreated\%20Children.pdf

Ronnestad, M. H., \& Skovholt, T. M. (2003). The journey of the counselor and therapist: Research findings and perspectives on professional development. Journal of Career Development, 30(1), 5-44. http://dx.doi.org/10.1177/089484530303000102

Rothschild, B. (2000). The body remembers. New York: W. W. Norton \& Company.

Scott, W. C., Kaiser, D., Othmer, S., \& Sideroff, S. I. (2005). Effects of an EEG biofeedback protocol on a mixed substance abusing population. American Journal of Drug \& Alcohol Abuse, 31, 455-469. http://dx.doi.org/10.1081/ada-200056807

Sexton, T. L., \& Whiston, S. C. (1994). The status of the counseling relationship: An empirical review, theoretical implications, and research directions. The Counseling Psychologist, 22, 6-78. http://dx.doi.org/10.1177/0011000094221002

Smith, W. (2008). The effect of neurofeedback training on PTSD symptoms of depression and attention problems among military veterans. (Doctoral dissertation). Retrieved from Proquest Dissertations and Theses database (UMI No. 3315214 )

Stokes, D. A., \& Lappin, M. S. (2010). Neurofeedback and biofeedback with 37 migraineurs: A clinical outcome study. Behavioral and Brain Functions 6:9. http://dx.doi.org/10.1186/1744-9081-6-9

Van der Kolk, B. A. (2003). The neurobiology of childhood trauma and abuse. Child and Adolescent Psychiatric Clinics of North America, 12, 293-317. http://dx.doi.org/10.1016/S1056-4993(03)00003-8

Van der Kolk, B. A. (2006). Clinical implications of neuroscience research in PTSD. New York Academy of Sciences, 1-17. http://dx.doi.org/10.1196/annals.1364.022 


\section{APPENDIX \\ Demographics Inventory \\ Neurofeedback Provider Demographic Information}

This form will be kept in a secure file by the researcher. Any information derived from it will be identified by a participant identification code only, in order to preserve your confidentiality.

\section{General Information:}
A. Name:
B. Name of practice:
C. Practice Address:
D. Phone number: Email address:

\section{Educational Background:}
A. In what field is your degree?
$\square$ Counseling
$\square$ Psychology
Social Work

$\square$ Psychiatry $\quad$ Other (please specify):

B. Highest degree completed: $\square$ Masters $\square$ Doctorate $\square$ Other

$\square$ Degree in progress (please specify):

(please specify):

C. Please list any licenses, certifications, or other special training that you have, and when you received/completed them:

III. Professional Experience:

A. Number of years in neurofeedback practice:

B. With which organization were you trained?

C. In addition to neurofeedback, do you also provide counseling/therapy services in your practice? $\quad$ Yes $\square$ No

D. If so, how many years have you been doing counseling/therapy?

IV. Client information:

A. Approximately how many clients have you seen per week, during this past year?

B. If possible, could you please give an estimate of the percentage of your clients with the following presenting problems:

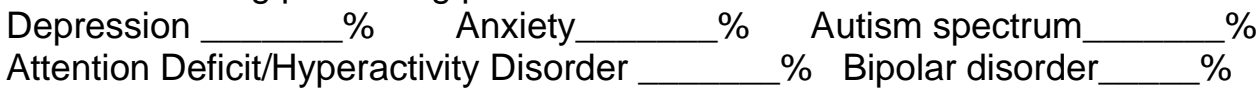
Chronic fatigue/fibromyalgia__ $\%$ Sleep problems $\quad \%$ Traumatic Brain Injury $\%$ Other common presenting problems:

C. What is the estimated percentage of your clients in which some form of trauma is a central issue: $\%$

[Including, but not limited to: Childhood abuse (physical, verbal, sexual), rape/sexual assault, domestic violence, combat experience, attachment traumas, physical injuries (accidents, etc.) to self or a loved one, etc.]

Please include any additional comments here, or on the back of this form, if needed:

Thank you very much for your participation in this research project! 


\section{Interview Protocol}

The initial interview question will be broad and general, in order to allow potentially unanticipated themes to emerge. Sub-questions will be more specific. In addition, the interview protocol may be altered, if themes emerge that need further exploration, or if the participant discusses sub-questions in the initial question.

I. Initial procedures:

A. Restate purpose of the study: You have been nominated by your peers as someone who is effective in providing neurofeedback services. I would like to explore your experiences and perceptions as a neurofeedback provider concerning the factors and processes that contribute to positive treatment outcomes when neurofeedback is used on individuals with trauma symptoms. Some form of traumatic experience is frequently an underlying factor in mental health issues such as depression, anxiety, substance abuse, self-destructive behaviors, relationship problems, and somatic symptoms. For this study, a wide definition of trauma will be used, which includes both the DSM-IV-TR criteria for posttraumatic stress disorder and the 7 symptoms associated with complex trauma (Courtois, 2008; Herman, 1992).

B. Make sure forms are signed:

$>$ Consent to Record interview

$>$ Informed Consent

C. Ask for (if not obtained already):

$>$ Completed Demographic Information Form

$>$ Resume/curriculum vitae (if available)

$>$ Brochures or materials which they typically give clients or use to describe their services; professional disclosure statement; articles, etc.

\section{Interview Protocol}

\section{Follow up on the Demographic Information Form:}

Is there any information that was not included on the Provider Demographic information form, that you would like to add?

\section{Opening questions:}

1. How did you first hear about neurofeedback?

a. What were your first impressions of neurofeedback?

2. What made you decide to set up a practice using neurofeedback, or to incorporate neurofeedback into your existing practice?

a. Is there anyone or anything in particular that influenced you or helped you?

3. What was it like when you first started using neurofeedback in your practice?

a. How is your practice different now from when you started?

4. Tell me what a typical day looks like for you in your practice.

a. Describe the typical clients who come to you for neurofeedback.

\section{Central Interview Questions}

You have been nominated by your peers as being effective in using neurofeedback to treat clients.

5. In your opinion, what factors have contributed to positive treatment outcomes when using neurofeedback in general? (factors: for example, personal, therapeutic, professional, therapeutic alliance, etc.)

a. In your opinion, what factors have contributed to positive treatment outcomes in using neurofeedback on clients with trauma symptoms?

b. Are there any factors that have been obstacles to successful treatment? 
c. How might multicultural factors contribute to positive or negative treatment outcomes?

(for example, gender, race, ethnicity, age, sexual orientation)

d. In your opinion, what factors distinguish an expert neurofeedback provider from a good provider, especially in treating individuals with trauma symptoms?

6. In your opinion, what part does years of experience play in a provider's expertise?

7. Now I would like to know more about the process of providing neurofeedback to clients who have experienced some type of trauma.

a. What types of symptoms have you noticed in clients who have experienced trauma?

b. Now that you've talked about , are there any additional symptoms? (Show list of PTSD symptoms and complex trauma symptoms)

c. Tell me about the process of treating those symptoms.

d. I'm wondering what differences you notice in treating clients who have experienced trauma, and those who haven't?

e. Are there any differences in your responses or feelings in treating these clients?

8. Now l'd like to ask you more about actual treatment. In general, how do you go about treating trauma symptoms?

a. I would like to hear about your experience treating a client with trauma symptoms, when the client seemed to be helped significantly.

b. How did you know the client was helped?

c. Now I would like to hear about an experience treating a client with trauma symptoms, when the client was helped very little, or not at all.

d. How did you know that the client was not helped?

e. In your opinion, what factors or processes contributed to the difference in the outcomes between the two clients?

Follow-up questions (for telephone interview):

9. What have been the most important lessons that you have learned through your Years of practice?

a. I would like to know about any critical incidents or circumstances that you have experienced, incidents that changed your views, or challenged you to do things a bit differently.

10. You've told me some factors, processes, and treatments that contribute to positive treatment outcomes. I'm wondering if you can narrow it down a bit. If you had to choose one thing that makes you successful in treating clients with trauma symptoms, what would it be?

11. In your opinion, how much of neurofeedback training is art versus science?

12. What have you noticed while treating clients with trauma symptoms, when neurofeedback is combined with counseling or some other treatment modality?

13. Tell me about your experiences using the alpha-theta protocol. (If not already discussed)

\section{Personal reflection questions:}

14. I'm wondering how you balance your professional practice with your personal life.

a. Do you ever take your clients home with you? (not physically but mentally and emotionally)

15. After having these experiences, what advice would you give to someone who wants to begin using neurofeedback as a treatment modality, particularly with those who have experienced trauma?

16. l'd like to know what you think about the future of neurofeedback, both personally and professionally.

a. Where do you see yourself in two years? Five years? Ten years? 
b. How will you be different then, both personally and professionally, from now? 17. Talk about the future of neurofeedback.
a. What are your thoughts about the future of neurofeedback?
b. What are your hopes for the future of neurofeedback?

\section{Email questions:}

18. Is there anything else that you would like to tell me about your experiences with neurofeedback?

19. If you could do it all over again (in terms of your mental health/neurofeedback career) what would you do the same?

a. What would you do differently? 